\title{
Carcinoma of Unknown Primary Site: Sequential Treatment with Paclitaxel/Carboplatin/Etoposide and Gemcitabine/Irinotecan: A Minnie Pearl Cancer Research Network Phase II Trial
}

\author{
F. Anthony Greco, ${ }^{a}$ Gladys I. Rodriguez, ${ }^{\text {b Don W. Shaffer }},{ }^{\mathrm{c}}$ Robert Hermann, \\ Sharlene Litchy, ${ }^{a}$ Denise A. Yardley, ${ }^{a}$ Howard A. Burris, III, ${ }^{a}$ Lisa H. Morrissey, ${ }^{a}$ \\ JoAn B. ERLAND, ${ }^{a}$ John D. HAInSWORTHa
}

The Sarah Cannon Cancer Center and Tennessee Oncology, PLLC, Nashville, Tennessee, USA;

bSouth Texas Oncology and Hematology, PA, San Antonio, Texas, USA; 'Northwest Georgia

Oncology Centers, PC, Marietta, Georgia, USA

Key Words. Carcinoma $\cdot$ Unknown $\cdot$ Chemotherapy $\cdot$ Sequential $\cdot$ Metastasis $\cdot$ Survival

\begin{abstract}
Purpose. To evaluate the efficacy and toxicity of the sequential administration of paclitaxel $\left(\right.$ Taxol $^{\circledR}$; BristolMyers Squibb; Princeton, NJ), carboplatin (Paraplatin ${ }^{\circledR}$; Bristol-Myers Squibb), and oral etoposide (VePesid ${ }^{\circledR}$; Bristol-Myers Squibb) followed by gemcitabine (Gemzar ${ }^{\circledR}$; Eli Lilly; Indianapolis, IN) and irinotecan (Campostar ${ }^{\circledR}$; Pfizer Pharmaceuticals; New York, NY) in the first-line treatment of patients with carcinoma of unknown primary site.

Patients and Methods. One hundred thirty-two patients were treated with sequential combination chemotherapy for a maximum of six cycles. All patients had relatively poor prognostic features. Fifty-nine patients had well-differentiated adenocarcinoma, 73 patients had poorly differentiated carcinoma, and 121 patients had performance status scores of 0 or 1.

Results. Thirty-three (30\%) of 111 assessable patients (95\% confidence interval $27 \%-33 \%$ ) had objective responses to treatment ( 26 partial responses, seven complete responses). The combination of gemcitabine and

irinotecan was associated with significantly less toxicity than the triple-drug regimen and improved the responses in several patients $(\mathbf{1 0 \%})$. The response rates were similar in the two major histologic tumor types, but were lower for patients with liver-dominant tumors $(13 \%)$ and higher for patients with lymph-node-dominant tumors $(\mathbf{5 0 \%})$. The median progression-free survival time, median survival time, and actuarial survival rates at 1 and 2 years were 5.7 months, 9.1 months, $35 \%$, and $16 \%$, respectively.

Conclusions. Sequential combination chemotherapy with paclitaxel/carboplatin/oral etoposide and gemcitabine/irinotecan is an active treatment for patients with carcinoma of unknown primary site, but overall toxicities are greater than those seen with other combinations of new drugs and survival appears similar to that observed in 264 other patients treated in our four previous phase II trials. A better understanding of the biology of these heterogeneous tumors will likely lead to improved therapy for these patients. The Oncologist 2004;9:644-652
\end{abstract}

\section{INTRODUCTION}

Since 1995, The Minnie Pearl Cancer Research Network (MPCRN) has performed five sequential phase II studies in a

total of 396 patients with unknown primary carcinoma using several new chemotherapy combinations. Initially, paclitaxel $\left(\right.$ Taxol ${ }^{\circledR}$; Bristol-Myers Squibb; Princeton, NJ) was tested in

Correspondence: F. Anthony Greco, M.D., Sarah Cannon Cancer Center, 250 25th Avenue North, Suite 110, Nashville, Tennessee 37203, USA. Telephone: 615-986-4300; Fax: 615-986-0029; e-mail: fgreco@tnonc.com Received April 26, 2004; accepted for publication July 8, 2004. (C)AlphaMed Press 1083-7159/2004/\$12.00/0 
combination with carboplatin (Paraplatin ${ }^{\circledR}$; Bristol-Myers Squibb), and oral etoposide (VePesid ${ }^{\circledR}$; Bristol-Myers Squibb) [1]; subsequently, docetaxel (Taxotere ${ }^{\circledR}$; Aventis Pharmaceuticals Inc.; Bridgewater, $\mathrm{NJ}$ ) was tested in combination with cisplatin (Platinol ${ }^{\circledR}$; Bristol-Myers Squibb) and later with carboplatin [2]. Patients with favorable prognostic features, known to be more treatable with better prognoses, were excluded from these studies. After a minimum follow-up of 4.5 years for the 144 patients in the first three studies, the median survival time was 10 months and the 1-, 2-, 3-, 4-, 5-, and 8-year survival rates were $42 \%, 22 \%, 18 \%, 16 \%, 12 \%$, and $10 \%$, respectively. Gemcitabine (Gemzar ${ }^{\circledR}$; Eli Lilly; Indianapolis, IN), a drug with activity in several solid tumors, was also found to be useful as secondary therapy for some patients with carcinoma of unknown primary site [3]. Subsequently, the combination of gemcitabine, carboplatin, and paclitaxel in the fourth study in 120 patients was found to be active, to be associated with acceptable toxicity, and to result in survival similar to our previous three taxane-based regimens for these patients [4]. Recent clinical experience with irinotecan (Campostar ${ }^{\circledR}$; Pfizer Pharmaceuticals; New York, NY), both alone and combined with gemcitabine [5], has been encouraging and relatively well tolerated as second-line therapy.

Based upon these observations, we evaluated the feasibility and efficacy of first-line treatment with paclitaxel/carboplatin/ etoposide followed by gemcitabine/irinotecan in patients with carcinoma of unknown primary site. We report here the results of this multicenter phase II study, conducted in the MPCRN.

\section{Patients And Methods}

\section{Eligibility}

Patients who had histologically confirmed metastatic carcinomas were eligible for the current study if the following evaluations had not revealed a primary site: complete history, physical examination, chemistry profile, chest X-ray, computed tomography of the chest, abdomen, and pelvis, mammography (in women), and directed work-up of any symptomatic areas. Patients were eligible who had light microscopic pathologic diagnoses as follows: well-differentiated adenocarcinoma, poorly differentiated adenocarcinoma (PDA), and poorly differentiated carcinoma (PDC). Serum markers were not required to enter patients in the trial, except $\beta$-human chorionic gonadotropin, and $\alpha$-fetoprotein, in those with PDC, and prostate-specific antigen (in men).

Patient subsets known to have relatively good prognostic features were excluded from this study. These subsets include women with adenocarcinoma involving only axillary lymph nodes or the peritoneal cavity; patients with squamous cell carcinomas involving only cervical or inguinal lymph nodes; patients with poorly differentiated carcinomas consistent with a germ cell tumor (i.e., isolated midline adenopathy/masses and/or multiple pulmonary nodules and/or elevated levels of $\beta$-human chorionic gonadotropin or $\alpha$-fetoprotein), men with prostate-specific antigen elevated in their plasma or stained in their tumors, patients with single potentially resectable tumors, patients with neuroendocrine carcinomas, and patients with poorly differentiated neoplasms, not otherwise classified.

A specific pathologic study to exclude other neoplasms was required for each patient with the initial diagnosis of PDC. Immunoperoxidase staining for leukocyte common antigen was required in all patients to exclude lymphoma. Additional immunoperoxidase stains, including epithelial markers (cytokeratins), neuroendocrine markers (synaptophysin, chromogranin), and melanoma markers (S-100, Hmb-45) were also performed routinely. For men with adenocarcinoma, staining for prostate-specific antigen was performed when clinical features suggested prostate carcinoma. Patients with specific neoplasms identified by special stains were excluded. The pathology reports were reviewed on every patient by two of the authors (F.A.G. and J.D.H.), but no central pathology review was done.

Previous chemotherapy was not allowed. Other entry criteria included an Eastern Cooperative Oncology Group (ECOG) performance status score of 0,1 , or 2 , adequate bone marrow function (white blood cells $\geq 4,000 / \mu 1$; platelets $\geq 100,000 / \mu \mathrm{l}$ ), normal liver function (bilirubin $\leq 1.5 \mathrm{mg} / \mathrm{dl}$ ), and normal renal function (creatinine $\leq 1.5 \mathrm{mg} / \mathrm{dl}$ ). Patients were excluded if they had any features as follows: recent myocardial infarction, congestive heart failure, other severe coexistent medical illnesses, a history of previous malignancy within 5 years with the exception of skin carcinoma or cervical carcinoma in situ, pregnant or lactating, and brain or meningeal metastases. The current study was approved by the appropriate Institutional Review Boards, and patients were required to give written informed consent before they participated in the study.

\section{Treatment}

The treatment received by patients and evaluation schedule in this trial are outlined in Table 1. All patients began treatment with two courses of paclitaxel/carboplatin/etoposide (regimen A) as follows: paclitaxel, $200 \mathrm{mg} / \mathrm{m}^{2}$, 1-hour i.v. infusion, day 1; carboplatin, at area under the concentration-time curve (AUC) of 6.0, i.v. infusion, day 1; and etoposide, $50 \mathrm{mg}$ (total dose) alternating with $100 \mathrm{mg}$ (total dose) orally daily, days $1-10$. Treatment courses were repeated at 21-day intervals. All patients received premedication with i.v. dexamethasone (Decadron ${ }^{\circledR}$; Merck and Company, Inc.; West Point, PA), $20 \mathrm{mg}$, diphenhydramine (Benadryl ${ }^{\circledR}$; Pfizer Pharmaceuticals; New York, NY), $50 \mathrm{mg}$, and cimetidine 
Table 1. Chemotherapy and reevaluation schema

Cycle 1,2-regimen A

$\begin{array}{lll}\text { Paclitaxel } & \text { Carboplatin } & \text { Oral etoposide } \\ 200 \mathrm{mg} / \mathrm{m}^{2} \text { i.v. } & (\text { AUC }=6) \text { i.v. } & 50 \mathrm{mg} \text { (total dose) } / 100 \mathrm{mg} \\ \text { day } 1 & \text { day } 1 & \text { (total dose) alternating } \\ \text { Repeat in } 3 \text { weeks (day 22) } & \text { daily } \times 10 \text { days } \\ \text { Cytokine use after cycle } 1 \text { at investigator discretion }\end{array}$

Reevaluate week 6; cycle 3,4-regimen B

$\begin{array}{ll}\text { Gemcitabine } & \text { Irinotecan } \\ 1,000 \mathrm{mg} / \mathrm{m}^{2} \text { i.v. } & 100 \mathrm{mg} / \mathrm{m}^{2} \text { i.v. } \\ \text { day } 1,8 & \text { day } 1,8\end{array}$

Repeat in 3 weeks (day 22)

Reevaluate week 12; cycle 5, 6

Options (investigator discretion):

give either regimen A or B for two cycles (every 3 weeks)

or give one cycle of each regimen (every 3 weeks)

\section{Reevaluate week 15}

Stop chemotherapy after a total of six cycles

Clinical follow-up every 2 months (reevaluation as clinically indicated)

(Tagamet $^{\circledR}$; GlaxoSmithKline; Research Triangle Park, NC), $300 \mathrm{mg}$, given 30 minutes before paclitaxel administration. The carboplatin dose was calculated by the Calvert formula as follows: dose $=([$ glomerular filtration rate +25$] \times 5)$. The glomerular filtration rate (GFR) was calculated by the Cockroft and Gault method using the following serum creatinine measurement: GFR $=([140-$ age $] \times$ weight in $\mathrm{kg}) \div$ $(72 \times$ serum creatinine) $\times$ either 0.85 (females) or 1.00 (males).

After completing two courses of regimen A, patients were reevaluated for response to treatment. Regardless of response, all patients then received two courses of gemcitabine/irinotecan (regimen B) as follows: gemcitabine, $1,000 \mathrm{mg} / \mathrm{m}^{2}$ i.v., days 1 and 8 ; and irinotecan, $100 \mathrm{mg} / \mathrm{m}^{2}$ i.v., days 1 and 8 ; treatment cycle repeated on day 22 .

After completing two cycles of regimen B, patients were once again reevaluated for response (week 12). At that time, patients with objective responses or stable disease received two additional courses of treatment. Selection of the treatment regimen for the fifth and sixth courses was at the discretion of the treating physician, based on the relative efficacy and toxicity of regimen A versus regimen B during the first four courses of treatment. In general, if one regimen appeared more efficacious or better tolerated, it was selected for the fifth and sixth courses of treatment. If the two regimens were similar, one additional course of each regimen was recommended. Patients with progressive disease after four courses were removed from study and treated at the discretion of their physician. All chemotherapy was stopped after a total of six cycles. Patients were reevaluated for a final time at week 15 and then observed off therapy and reevaluated as clinically indicated.

During therapy, blood counts were monitored weekly. Dose modifications for myelosuppression were based on blood counts measured on the day of scheduled treatment. The day 1 dose modifications for regimen A (paclitaxel/carboplatin/oral etoposide) were as follows: absolute neutrophil count (ANC) $\geq 1,500 / \mu 1$ and platelets $\geq 100,000 / \mu 1,100 \%$ doses; ANC $1,000-1,500 / \mu 1$ or platelets $75,000-100,000 / \mu 1$, $75 \%$ doses; ANC $<1,000 / \mu 1$ or platelets $<75,000 / \mu 1$, treatment delay for 1 week or until ANC and platelets rose above these levels, then $75 \%$ doses. If there was a 3-week delay, the patient was taken off study. On day 8 , if the ANC was $\geq 1,500 / \mu 1$ and the platelet count was $\geq 75,000 / \mu 1$, the 10-day course of oral etoposide was completed. If the respective ANC and platelet counts were less than as stated, the remaining etoposide (i.e., days 8-10) was omitted. Any hospitalization for neutropenia and fever dictated a $25 \%$ dose reduction of paclitaxel and carboplatin and a reduction in the dose of oral etoposide to $50 \mathrm{mg}$ total dose daily. Reversible grade $3 / 4$ nonhematologic toxicities, with the exception of nausea and vomiting and alopecia, required a $25 \%$ dose reduction of the responsible agent or agents. If grade $3 / 4$ nonhematologic toxicity was irreversible or had not resolved to $\leq$ grade 1 after a treatment delay of 3 weeks, the patient was removed from study. Patients who experienced severe (grade 3 or 4 ) acute hypersensitivity reactions related to either paclitaxel or carboplatin were removed from the study.

For regimen B (irinotecan/gemcitabine), dose modifications on days of scheduled treatment were as follows: ANC $>1,500 / \mu 1$ and platelets $>100,000 / \mu 1,100 \%$ doses; ANC $1,000-1,500 / \mu 1$ or platelets $75,000-100,000 / \mu 1,75 \%$ doses; ANC $<1,000 / \mu 1$ or platelets $<75,000 / \mu 1$, dose delay for 1 week or until ANC $>1,000 / \mu 1$ and platelets $>100,000 / \mu 1$, then further doses at $75 \%$ for both drugs. If more than a 3week delay was required, the patient was taken off study and treated at the discretion of their physicians. Hospitalization for neutropenia and fever required a $25 \%$ dose reduction of both drugs for all remaining doses. Patients with reversible grade $3 / 4$ nonhematologic toxicities had $25 \%$ dose reductions of the offending agent(s). Prolonged grade $3 / 4$ nonhematologic toxicities for 3 weeks required that the patient be taken off study. For diarrhea, standard prophylactic loperamide (Imodium ${ }^{\circledR}$; McNeil Consumer and Specialty Pharmaceuticals; Fort Washington, PA) was administered for grade 1 or 2. For grade 3 or 4 diarrhea, irinotecan was withheld and intensive therapy for diarrhea was initiated; irinotecan was resumed at the $75 \%$ dose when diarrhea improved to grade 1 or less. 
Cytokines were not used prophylactically during the first course of treatment for any patient. Subsequent use of cytokines was at the discretion of the treating physician. In particular, if it was deemed important to continue treatment on schedule in a patient who had demonstrated an early response to therapy, cytokines could be used prophylactically to maintain dose and schedule.

\section{Assessment of Treatment Efficacy}

Patients were evaluated for tumor response three times during treatment (Table 1). These evaluations occurred after completing two courses of regimen $\mathrm{A}$ (paclitaxel/carboplatin/oral etoposide), after completing two courses of regimen B (irinotecan/gemcitabine), and at the completion of all treatment (six courses). At each reevaluation, a response category was assigned based on standard World Health Organization (WHO) definitions. The complete response category required the total disappearance of all clinically and radiologically detectable disease for at least 4 weeks. The partial response category required at least a $50 \%$ reduction in the size of all measurable lesions as measured by the product of the greatest length and the greatest width, with no new lesions appearing. Patients in the stable disease category had reductions of $<50 \%$ or increases of $<25 \%$ in the size of measurable lesions, with no new lesions appearing. Patients assigned to the progressive disease category had development of any new lesions or an increase of $25 \%$ or more in the size of any existing measurable lesion.

All patients who received at least one dose of treatment were included in the evaluation of treatment-related toxicity. Toxicities were graded according to the National Cancer Institute Common Toxicity Criteria, Version 2.0.

\section{Statistical Considerations}

The primary goals of the present study were to determine the toxicity, objective tumor response rate, progression-free survival (PFS) duration, and overall survival duration in patients receiving the sequential administration of paclitaxel/carboplatin/etoposide and irinotecan/gemcitabine. It remains difficult to determine the number of patients necessary for a phase II trial involving carcinoma of unknown primary site. Most accrual designs for phase II trials evaluate patients with relatively homogeneous neoplasms. In contrast, carcinoma of unknown primary site demonstrates extreme histologic and clinical heterogeneity, often with variable responsiveness to cytotoxic chemotherapy. Therefore, we took the liberty of arbitrarily adding a total of 80 patients to the two-staged ( 20 patients in stage 1 and 60 patients in stage 2) accrual design method of Simon [6]. We estimated this number would dilute the problem of extreme heterogeneity and variable responsiveness to cytotoxic therapy in this patient group to some degree and provide a better estimation of the toxicity, objective response rate, and PFS time. We expected at least a $20 \%$ response rate, and if fewer than eight of the first 40 patients entered in this trial (stage 1) failed to obtain objective responses, the study would end. If eight or more responses were seen, an additional 90 patients (stage 2) would be accrued. A secondary objective was to determine survival following therapy.

All patients were followed up until the time of progression or death. PFS was defined as the interval from the first day of therapy until tumor progression was documented. Actuarial PFS and survival curves were constructed using the Kaplan and Meier method [7].

All patient data (including copies of all primary source documents) were reviewed at the data center of the MPCRN at the Sarah Cannon Cancer Center. All response and toxicity validations were done by reviewing primary source documents.

\section{RESUlts}

\section{Patient Characteristics}

One hundred thirty-two patients were treated in the present study between December 2000 and September 2002. Patient characteristics are listed in Table 2 . The majority of patients had good performance status scores; $68 \%$ had either adenocarcinoma or PDA. Most patients (76\%) had metastases in one or more visceral sites; however, $24 \%$ had dominant tumor sites in lymph nodes or soft tissue. Patients were treated at the Sarah Cannon Cancer Center or by participating affiliate members of the MPCRN (Appendix 1).

\section{Treatment Received}

One hundred eleven of 132 patients (84\%) were evaluable for tumor response after two cycles of regimen A. Twenty-one patients did not receive two cycles of chemotherapy and were not evaluable for response after regimen A for the following reasons: severe paclitaxel hypersensitivity (four patients); other severe toxicity (six patients); patient refusal (four patients); intercurrent unrelated illness (two patients); and treatment-related deaths (five patients). Ninety-three patients subsequently received regimen B. Eighteen of the 111 evaluable patients after regimen A did not receive regimen $\mathrm{B}$ for the following reasons: progressive tumor and worsening performance status (11 patients); toxicities from regimen A precluding further chemotherapy (five patients); and patient refusal (two patients). Sixty-five patients started on cycles 5 and 6 as planned. Twenty-eight of the 93 patients that received regimen $B$ did not start on the planned cycles 5 and 6 for the following reasons: progressive tumor 


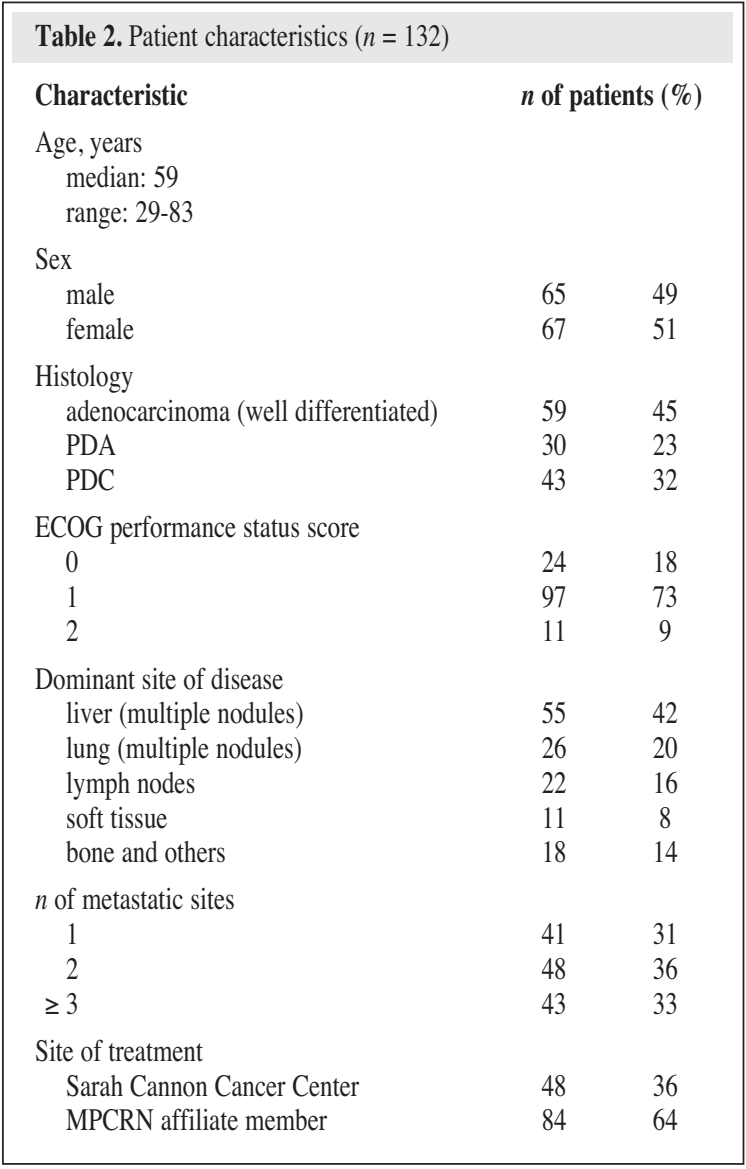

(25 patients); prolonged toxicity (three patients); and patient refusal (two patients). The best overall response data were calculated with 111 patients as the denominator.

\section{Efficacy}

In the first stage of this trial, in 40 assessable patients, nine attained objective responses $(22 \%)$ and the second stage of this study was initiated. Twenty-one of 111 patients (19\%) had objective tumor responses after two cycles of regimen A (19 partial responses, two complete responses). Sixty-eight patients remained stable and 22 patients had progressive tumors.

Regimen B was delivered to 93 patients. Ten (10\%) additional objective responses were seen, including eight partial responses (all stable after regimen A) and two complete responses (both stable after regimen A). Forty-two patients remained stable, and 25 patients had progressive tumors. Of the 11 patients with progressive tumors after regimen $\mathrm{A}$ who received regimen $\mathrm{B}$, none had an objective response.

A total of 65 patients began cycles 5 and 6 with an option of two cycles of regimen A, two cycles of regimen $\mathrm{B}$, or one cycle of each regimen. There were four patients showing additional improvements in tumor responses, including one partial response (stable after regimen B) and

\begin{tabular}{|c|c|}
\hline Tennessee Oncology, PLLC & Nashville, TN \\
\hline South Texas Oncology and Hematology & San Antonio, TX \\
\hline Northwest Georgia Oncology Centers & Marietta, GA \\
\hline Comprehensive Cancer Institute & Huntsville, AL \\
\hline $\begin{array}{l}\text { Oncology/Hematology Group of } \\
\text { South Florida }\end{array}$ & Miami, FL \\
\hline Atlanta Cancer Care & Atlanta, GA \\
\hline $\begin{array}{l}\text { Consultants in Blood Disorders } \\
\text { and Cancer }\end{array}$ & Louisville, KY \\
\hline Graves-Gilbert Clinic & Bowling Green, KY \\
\hline Mary Bird Perkins Cancer Center & Baton Rouge, LA \\
\hline Louisiana Oncology Associates & Lafayette, LA \\
\hline Grand Rapids CCOP & Grand Rapids, MI \\
\hline The Medical Oncology Group & Gulfport, MS \\
\hline Upstate Carolina CCOP & Spartanburg, SC \\
\hline Greenview Regional Hospital & Bowling Green, KY \\
\hline Northeast Georgia Medical Center & Gainesville, GA \\
\hline Jackson Oncology Associates & Jackson, MS \\
\hline The Hematology and Oncology Clinic & Hattiesburg, MS \\
\hline $\begin{array}{l}\text { Northeast Alabama Regional Medical } \\
\text { Center }\end{array}$ & Annison, $\mathrm{AL}$ \\
\hline Medical Oncology, LLC & Baton Rouge, LA \\
\hline $\begin{array}{l}\text { New Mexico Oncology/Hematology } \\
\text { Consultants }\end{array}$ & Albuquerque, NM \\
\hline Hematology and Oncology Services & New Orleans, LA \\
\hline Cancer Outreach Associates, PC & Abingdon, VA \\
\hline The Reading Hospital and Medical Center & West Reading, PA \\
\hline Northwest Oncology and Hematology & Elk Grove Village, IL \\
\hline Thompson Oncology Group & Knoxville, TN \\
\hline $\begin{array}{l}\text { University of Tennessee Cancer } \\
\text { Institute/Boston Baskin Cancer Group }\end{array}$ & Memphis, TN \\
\hline $\begin{array}{l}\text { Oncology/Hematology Associates of } \\
\text { SW Indiana }\end{array}$ & Evansville, IN \\
\hline St. Joseph Mercy Hospital Cancer Center & Pontiac, MI \\
\hline University Oncology & Chattanooga, TN \\
\hline $\begin{array}{l}\text { Central Georgia Hematology Oncology } \\
\text { Associates }\end{array}$ & Macon, GA \\
\hline $\begin{array}{l}\text { Kingsport Hematology and Oncology } \\
\text { Associates }\end{array}$ & Kingsport, TN \\
\hline St. Vincent Hospital Cancer Center & Green Bay, WI \\
\hline St. Louis Cancer Care & Chesterfield, MO \\
\hline Columbia Oncology & Columbia, TN \\
\hline
\end{tabular}

three complete responses (one stable, two partial responses after regimen B). Thirty-nine patients remained stable and one patient had progressive tumor.

The best overall responses during therapy for the 111 evaluable patients were as follows: 33 major responses (30\%), including $26(23 \%)$ partial responses and seven 
(7\%) complete responses. Thirty-five patients (31\%) had stable disease as their best response, and 43 (39\%) had progressive disease (Table 3). The response duration ranged from 3-33+ months (median $=7$ months).

As noted with other empiric therapies for patients with carcinoma of unknown primary site, the response rates varied depending on the site of predominant tumor involvement (Table 3). Patients with predominantly lymph node tumors had a 50\% response rate, while those with liver metastases had a response rate of only $13 \%$. There were no differences in response rate based on tumor histology (i.e., adenocarcinoma versus PDC).

All 132 patients were included in the survival analysis. The PFS analysis is illustrated in Figure 1. The median PFS duration was 5.2 months ( $95 \%$ confidence interval $[\mathrm{CI}]=4.4$ 6.0), with a 1-year PFS rate of $16 \%$ and a 2-year PFS rate of $6 \%$. The actuarial survival curve for all patients is shown in

\begin{tabular}{|lrl|}
\hline Table 3. Best response to therapy & & \\
Response & $\boldsymbol{n}$ of patients (\%) \\
Overall & $33^{*}(30 \%)$ \\
Complete & 7 & $(7 \%)$ \\
Partial & 26 & $(23 \%)$ \\
Stable/minor & 35 & $(25 \%)$ \\
Progression & $43(31 \%)$ \\
Inevaluable & $21(14 \%)$ \\
Responses by dominant tumor location & & \\
Liver & 7 & $(13 \%)$ \\
Lung & 8 & $(31 \%)$ \\
Lymph node & 11 & $(50 \%)$ \\
Soft tissue & 2 & $(18 \%)$ \\
Bone/others & 5 & $(18 \%)$ \\
\hline *95\% CI = 27\%-33\% & & \\
\hline
\end{tabular}

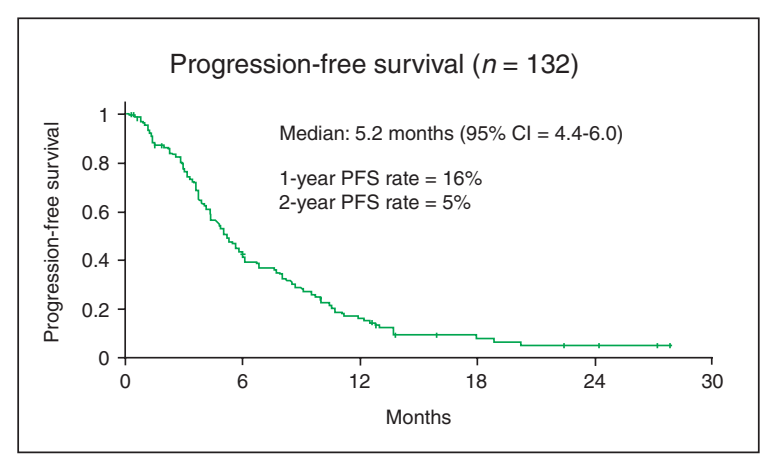

Figure 1. The median duration of PFS of the 132 patients was 5.2 months, with a 1-year PFS rate of $16 \%$ and a 2 -year PFS rate of $6 \%$.
Figure 2. The minimum follow-up was 15 months (range 1536 months). The median survival duration was 9.1 months $(95 \% \mathrm{CI}=8.1-10.2)$, with a 1-year survival rate of $35 \%$ and 2 -year survival rate of $16 \%$. Figure 3 compares the survival analysis for patients treated in this study with the updated survival analysis for the 264 patients reported in our previous four sequential phase II trials of taxane- or gemcitabine-based chemotherapy [1-4]. There is no significant difference (log rank $p=0.2361$ ) in these curves. For the previous 264 patients in four trials, the minimum follow-up was 30 months (range 30-96 months) and the median survival duration was 9.2 months, with survival rates at 1,2,3,5, and 8 years of $38 \%$, $19 \%, 16 \%, 10 \%$, and $8 \%$, respectively. The PFS analysis from those four trials is compared with the analysis from this study in Figure 4, and the results of these two analyses also do not appear to differ ( $\log \operatorname{rank} p=0.6836)$.

\section{Toxicity}

All 132 patients were assessed for toxicity. Table 4 outlines the grade 3 and 4 toxicities seen with regimen A

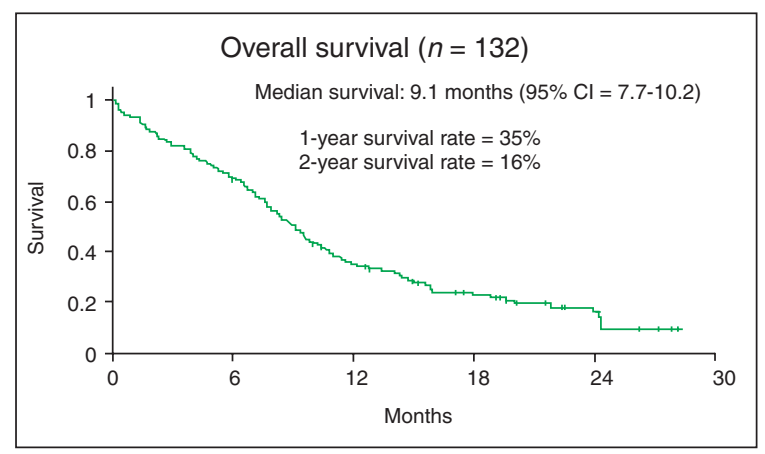

Figure 2. The median duration of survival of the 132 patients was 9.1 months, with a 1-year survival rate of $35 \%$ and 2-year survival rate of $16 \%$.

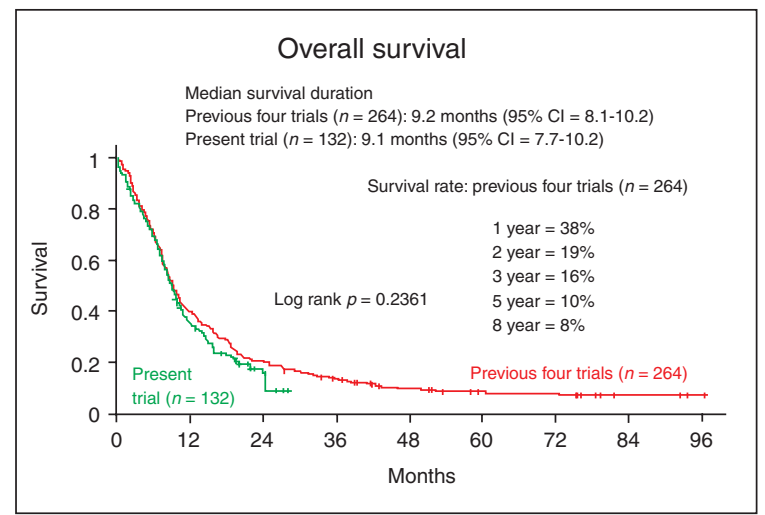

Figure 3. A comparison is illustrated of the survival analyses of the 132 patients in this study and of the 264 patients in four previous studies $(\log$ rank $\mathrm{p}=0.2361)$. 


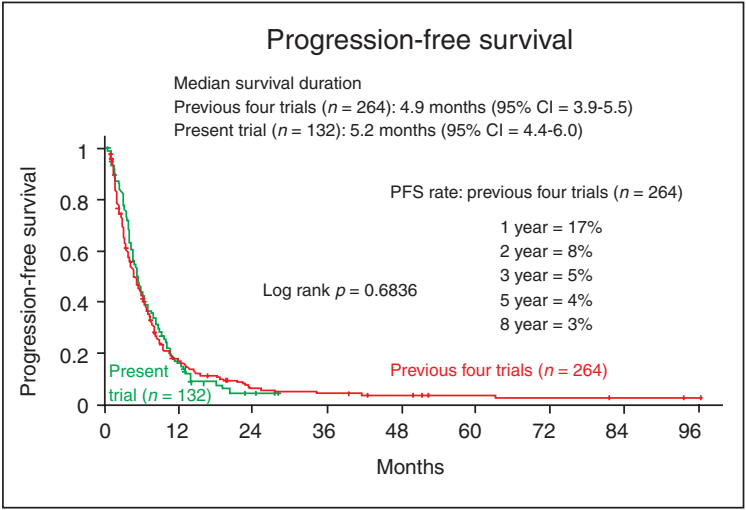

Figure 4. A comparison is illustrated of the PFS analyses of the 132 patients in this study and of the 264 patients in four previous studies (log rank $\mathrm{p}=0.6836)$.

(paclitaxel/carboplatin/etoposide) and regimen B (gemcitabine/ irinotecan). A total of 583 courses of chemotherapy was administered, including 307 courses of regimen A and 276 courses of regimen B. Regimen A was more toxic, and myelosuppression represented the major toxicity. Grade 3/4 leukopenia was seen in $71 \%$ of patients receiving regimen $\mathrm{A}$ versus $28 \%$ of those receiving regimen B $(p=0.001)$. Fever and neutropenia requiring hospitalization were seen in 17 patients on regimen $\mathrm{A}(13 \%)$ and in only one patient $(1 \%)$ on regimen $\mathrm{B}(p=0.001)$. Five treatment-related deaths were seen on regimen A and none were seen on Regimen B. Documented infections were seen in 19 patients (14\%) on regimen A and in five patients (5\%) on regimen B $(p=0.01)$. Other nonhematologic toxicities were fairly similar between the two regimens, with the exceptions of a significantly greater incidence of diarrhea on regimen B $(p=0.01)$ and less arthralgia/myalgia on regimen $\mathrm{B}(p=0.01)$.

\section{Discussion}

Combination sequential chemotherapy with paclitaxel, carboplatin, and oral etoposide followed by gemcitabine and irinotecan proved to be an active and useful therapy for some patients with unknown primary carcinomas. It is difficult to precisely ascribe the contribution of the therapies of this sequential approach. However, it is clear that the combination of gemcitabine and irinotecan is less toxic than the triple-drug regimen of paclitaxel, carboplatin, and etoposide. Furthermore, several additional objective responses were documented following sequential therapy with gemcitabine and irinotecan, suggesting some degree of noncrossresistant activity of this regimen for patients initially receiving paclitaxel, carboplatin, and oral etoposide.

The overall response rate seen in this sequential phase II trial was 30\%, with 33 responses, including 26 partial responses and seven complete responses. Responses varied according to the dominant site of metastatic disease (Table 3 ), and about half the evaluable patients had liver-dominant disease, a site known to be associated with lower response rates and a relatively poor prognosis [8]. The overall PFS duration and survival duration of the 132 patients entered on this large phase II trial were very similar to those found in our previous four phase II trials in a total of 264 patients [1-4] treated with taxanes combined with either carboplatin or cisplatin and with etoposide or gemcitabine (Fig. 3 and Fig. 4). The median survival time (9 months), 1-year survival rate (38\%), and 2-year survival rate (19\%) were

\begin{tabular}{|c|c|c|c|c|}
\hline \multirow[b]{2}{*}{ Toxicity } & \multicolumn{2}{|c|}{ Regimen A-grade $3 / 4$} & \multicolumn{2}{|c|}{ Regimen B-grade $3 / 4$} \\
\hline & $n$ of patients $(n=132)$ & $\%$ & $n$ of patients $(n=93)$ & $\%$ \\
\hline \multicolumn{5}{|l|}{ Hematologic } \\
\hline Leukopenia & 94 & 71 & 28 & $20(p=0.001)$ \\
\hline Thrombocytopenia & 19 & 13 & 22 & 23 \\
\hline Anemia & 14 & 11 & 5 & $5(p=0.01)$ \\
\hline Hospitalizations, fever/neutropenia & 17 & 13 & 1 & $1(p=0.001)$ \\
\hline Treatment-related death (sepsis) & 5 & 4 & 0 & 0 \\
\hline \multicolumn{5}{|l|}{ Nonhematologic } \\
\hline Fatigue & 12 & 9 & 11 & 12 \\
\hline Diarrhea & 7 & 4 & 11 & $12(p=0.01)$ \\
\hline Arthralgia/myalgia & 7 & 4 & 1 & $1(p=0.01)$ \\
\hline Peripheral neuropathy & 2 & 2 & 0 & 0 \\
\hline Nausea/vomiting & 14 & 11 & 9 & 9 \\
\hline Hypersensitivity & 4 & 3 & 0 & 0 \\
\hline Infection & 19 & 14 & 5 & $5(p=0.01)$ \\
\hline
\end{tabular}


similar to those seen in the 132 patients reported here. Furthermore, when considering the previous 264 patients, long-term survival was seen at 3, 4, 5, 6, and 8 years (Fig. 3). A smaller number of patients were alive at these time frames free of any known progressive tumor (Fig. 4). Follow-up for more than 1 year is not usually reported in prospective studies of unknown primary carcinoma patients; therefore, the frequency of PFS for more than 1 year with other regimens cannot be assessed.

These survival statistics illustrate the problems of comparing response rates and median survival times as measures of efficacy in various studies. Median survival times have not changed very much (a few months) from those resulting from chemotherapy given more than a decade ago. However, newer chemotherapy regimens are associated with a minority of the patients surviving for several years. Survival beyond 1 year was very rarely reported in prospective studies in the past. The survival rates at 1, 2, and 3 years and beyond are essential in comparing therapies for these patients, and in making judgments concerning the efficacy of therapy.

Several other phase II studies of the newer cytotoxic drugs, including paclitaxel, gemcitabine, and irinotecan in combination with a platinum agent, have also been reported [9-14]. Those studies showed similar response rates and median survival durations as reported here. However, longterm follow-up has generally not been reported. The definitive comparison of various therapies requires phase III prospective randomized trials.

Even though the phase II trial reported here was large, there are still many subgroups of patients represented with varying clinical and pathologic features and responsiveness to cytotoxic therapy. Prognostic factors in these patients are slowly coming to light $[11,14]$, and it will be necessary in the future to further stratify, or separate, patients with varying prognoses. Several subsets of patients with known good prognostic features were excluded from this trial and our previous four trials. However, other subgroups of patients with different prognoses were included (i.e., those with lymph-node-dominant and liver-dominant tumors).

The overall response rate, PFS time, and survival duration resulting from the sequential approach reported here do not appear to be any better than those previously reported with other combinations of new cytotoxic drugs [9-14]. Furthermore, the overall toxicity of the sequential administration of these combinations was greater than that seen in our previous four studies as well as in other reports [9-14]. Based upon the long-term survival rates observed in the 396 patients in our five phase II trials, a very compelling argument can be made that incorporating newer cytotoxic drugs (paclitaxel, docetaxel, gemcitabine, irinotecan) into therapy results in superior survival. We are now conducting a randomized phase III prospective trial comparing paclitaxel/carboplatin/etoposide with gemcitabine/irinotecan in previously untreated patients.

We expect the treatment of patients with carcinoma of unknown primary site to be less empiric and more effective once the lineage and biologic features of these tumors are better understood. Gene-expression profiling has particular promise as a means to determine the primary site, and perhaps the biology of individual tumors, and may also provide clues to more specific targeted therapies.

\section{ACKNOWLEDGMENTS}

Supported in part by grants from the Minnie Pearl Cancer Foundation, Bristol-Myers Squibb, Eli Lilly, and Pfizer.

\section{REFERENCES}

1 Hainsworth JD, Erland JB, Kalman LA et al. Carcinoma of unknown primary site: treatment with 1-hour paclitaxel, carboplatin, and extended-schedule etoposide. J Clin Oncol 1997;15:2385-2393.

2 Greco FA, Erland JB, Morrissey LH et al. Carcinoma of unknown primary site: phase II trials with docetaxel plus cisplatin or carboplatin. Ann Oncol 2000;11:211-215.

3 Hainsworth JD, Burris HA 3rd, Calvert SW et al. Gemcitabine in the second-line therapy of patients with carcinoma of unknown primary site: a phase II trial of the Minnie Pearl Cancer Research Network. Cancer Invest 2001;19:335-339.

4 Greco FA, Burris HA 3rd, Litchy S et al. Gemcitabine, carboplatin, and paclitaxel for patients with carcinoma of unknown primary site: a Minnie Pearl Cancer Research Network study. J Clin Oncol 2002;20:1651-1656.
5 Doss HH, Hainsworth JD, Spigel DR et al. Gemcitabine and irinotecan in patients with previously treated carcinoma of unknown primary site: a Minnie Pearl Cancer Research Network phase II trial. Proc Am Soc Clin Oncol 2004;23:4167.

6 Simon R. Optimal two-stage designs for phase II clinical trials. Control Clin Trials 1989;10:1-10.

7 Kaplan EL, Meier P. Non-parametric estimation from incomplete observations. J Am Stat Assoc 1958;53:457-481.

8 Hess KR, Abbruzzese MC, Lenzi R et al. Classification and regression free analysis of 1000 consecutive patients with unknown primary carcinoma. Clin Cancer Res 1999;5:34033410 .

9 Pavlidis N, Briasoulis E, Hainsworth J et al. Diagnostic and therapeutic management of cancer of an unknown primary. Eur J Cancer 2003;39:1990-2005. 
10 Pavlidis N, Kalofonos H, Bafaloukos D et al. Cisplatin/Taxol combination chemotherapy in 72 patients with metastatic cancer of unknown primary site: a phase II trial of the Hellenic Cooperative Oncology Group. Proc Am Soc Clin Oncol 1999;18:195a.

11 Briasoulis E, Kalofonos H, Bafaloukos D et al. Carboplatin plus paclitaxel in unknown primary carcinoma: a phase II Hellenic Cooperative Oncology Group study. J Clin Oncol 2000;18:3101-3107.

12 Lastra E, Munoz A, Rubio I et al. Paclitaxel, carboplatin, and oral etoposide in the treatment of patients with carcinoma of unknown primary site. Proc Am Soc Clin Oncol 2000;19:579a.

13 Mukai H, Watanabe T, Ando M et al. A safety and efficacy trial of docetaxel and cisplatin in patients with cancer of unknown primary. Proc Am Soc Clin Oncol 2000;22:646.

14 Culine S, Lortholary A, Voigt JJ et al. Cisplatin in combination with either gemcitabine or irinotecan in carcinomas of unknown primary site: results of a randomized phase II study - trial for the French Study Group on Carcinomas of Unknown Primary (GEFCAPI 01). J Clin Oncol 2003;21:3479-3482. 